BNL-96512-2011-IR

\title{
Options for Production Staging for a Low Energy Neutrino Factory
}

\author{
J. Scott Berg
}

October 2011

Physics Department

Brookhaven National Laboratory

\section{U.S. Department of Energy Office of Science}

Notice: This manuscript has been authored by employees of Brookhaven Science Associates, LLC under Contract No. DE-AC02-98CH10886 with the U.S. Department of Energy. The publisher by accepting the manuscript for publication acknowledges that the United States Government retains a non-exclusive, paid-up, irrevocable, world-wide license to publish or reproduce the published form of this manuscript, or allow others to do so, for United States Government purposes. 


\section{DISCLAIMER}

This report was prepared as an account of work sponsored by an agency of the United States Government. Neither the United States Government nor any agency thereof, nor any of their employees, nor any of their contractors, subcontractors, or their employees, makes any warranty, express or implied, or assumes any legal liability or responsibility for the accuracy, completeness, or any third party's use or the results of such use of any information, apparatus, product, or process disclosed, or represents that its use would not infringe privately owned rights. Reference herein to any specific commercial product, process, or service by trade name, trademark, manufacturer, or otherwise, does not necessarily constitute or imply its endorsement, recommendation, or favoring by the United States Government or any agency thereof or its contractors or subcontractors. The views and opinions of authors expressed herein do not necessarily state or reflect those of the United States Government or any agency thereof. 


\title{
Options for Production Staging for a Low Energy Neutrino Factory
}

\author{
J. Scott Berg \\ Brookhaven National Laboratory; PO BOX 5000; UPTON NY 11973-5000 \\ October 26, 2011
}

\begin{abstract}
A low energy neutrino factory (LENF) is defined, for the purpose of this report, to accelerate a muon beam to a total energy in the range of $10-14 \mathrm{GeV}$, and store it in a decay ring directing a resulting neutrino beam to a detector $2200-2300 \mathrm{~km}$ distant $[1,2]$. The machine should be ultimately capable of producing $10^{21}$ decays toward that detector per year of $10^{7} \mathrm{~s}$. We consider such a neutrino factory to be the accelerator defined in the Interim Design Report (IDR) of the International Design Study for the Neutrino Factory (IDS-NF) [3], modified to remove the final stage of acceleration, possibly modifying the remaining acceleration stages to adjust the final energy, and replacing the decay ring with one designed for the lower energy and shorter baseline. We discuss modifications to that design which would reduce the cost of the machine at the price of a reduction in neutrino production, down to as low as $10^{20}$ decays per year. These modifications will not preclude eventually upgrading the machine to the full production of $10^{21}$ decays per year. The eventual cost of a machine which achieves the full production through a series of lower-production stages should not exceed the cost of a machine which is immediately capable of the full production by more than a small fraction of the cost difference between the full production machine and the lowest production stage.
\end{abstract}

\section{Stages which can be Modified}

The first step is to examine the individual parts of the accelerator to determine which portions can potentially be modified to reduce their cost while reducing the eventual muon production. This examination is constrained to ensure that it will not be excessively costly to eventually construct a machine with the full neutrino production.

This manuscript has been authored by employees of Brookhaven Science Associates, LLC under Contract No. DE-AC02-98CH10886 with the U.S. Department of Energy. The United States Government retains a non-exclusive, paid-up, irrevocable, world-wide license to publish or reproduce the published form of this manuscript, or allow others to do so, for United States Government purposes.

\section{Proton Driver}

The proton driver is the most obvious place to reduce the machine cost. This can come in two ways: a reduction in the beam current, and a failure to meet the required beam specifications. For the latter, the bunch length could be longer than desired (production is maximal for $1 \mathrm{~ns}$ RMS and below, the IDR tolerates $3 \mathrm{~ns})$, or the transverse emittance could be larger (the IDR specifies $5 \mu \mathrm{m}$ RMS).

One could also lower the proton beam energy (5-15 GeV in the IDR), but that may impose significant costs for upgrading to full production. A bunched beam must be delivered to the target. For scenarios consisting of a linac followed by accumulator and compressor rings, the accumulator ring (which produces the bunches) must be designed for the proton 
energy that is delivered to the target. Changing that final energy will either require a new accumulator ring, or will require replacing most of the magnets in the pre-existing ring. Either scenario is likely to introduce significant additional costs.

One can imagine many ways in which the proton beam current could be reduced, I will not address most of them here. One might consider a reduction in repetition rate in particular, since that may allow a staging of the RF systems in the accumulator and compressor rings (for proton driver scenarios that have these).

One could have a significantly longer bunch delivered to the target [4], either by reducing the amount of RF in a compressor ring, or by adding sufficient RF voltage to the accumulator ring to provide the more modest bunching (or for scenarios with only rings, a reduction in the amount of RF in the final ring). One could imagine a bunch as long as around 20 ns RMS. Adding RF to the accumulator ring would increase the overall cost of the final system since the additional accumulator ring RF would be unnecessary in the full-performance machine, but this cost may be modest.

At Fermilab, one would expect to use the existing proton driver infrastructure to start with, then later one may use Project $X$, staging as described above.

\section{Target}

There are probably few options for cost reduction in the target. Any target station will need to be capable of handling the full beam power. If the proton driver repetition rate is reduced, one may be able to flow a liquid target more slowly, but eventually the system must be capable of the higher flow rate.

One could initially have a lower magnetic field in the target region. One would do this by initially omitting some of the magnets around the target, in particular the resistive coils, which in the current design are part of a removable target module. The resistive coil could be replaced with additional shielding to further reduce the cryogenic load on the superconducting magnets, thereby reducing the cost of the cryogenic plant.

One could also initially use a simpler target, such as a solid carbon target, assuming that the proton driver power is lower.

It would be possible to construct a separate target station at a different location, permitting a shorter front end (see below). This would probably introduce an unacceptable cost for the full performance system, since this target station would need to be completely replaced.

\section{Front End}

Assuming that the target station is in the same location as the target station for the full performance machine, the length of the front end beamline will need to be the same in the low-performance stages as it is in the fullperformance machine.

The magnetic lattice should be the same for the low-performance stages as it is for the fullperformance machine. The beam must be transported over the full length, and removing any magnets from the existing lattice would likely result in a total beam loss. One could rearrange magnets, but this would likely result in significant additional costs.

The best option for reducing costs in the front end is to install less RF voltage, by using less power in the RF cavities or by installing fewer RF cavities. This will decrease the energy acceptance of the front end, thereby reducing performance.

\section{Acceleration}

Since the primary goal of the acceleration system is to reach the energy for which the decay ring is designed, the acceleration system must essentially remain the same irrespective of the performance. Layout geometry also limits the options one has for acceleration. 


\section{Decay Ring}

The decay ring should be designed to store beam at the final energy. It is probably not possible to modify this.

\section{Work Plan}

To implement this plan, I would propose the following simulation studies:

1. Proton driver (1 FTE)
a. Reduced RF in compression ring to give longer bunch
b. Eliminate compression ring, add RF to accumulator ring to get longer bunch
c. Reduction in repetition rate

2. Target (0.5 FTE)
a. Effect of removing resistive coil
b. Reduction in cryogenic requirements from replacing resistive coil with shielding
c. Replacing mercury jet target with carbon or other solid target

3. Front end (1 FTE)
a. Reduction in RF voltage in bunching and phase rotation
b. Removal of absorbers from cooling
c. Reduction in RF voltage in (absorber- free) cooling

These simulations will need to determine the performance reductions that result from these changes. Then the cost benefit of these solutions will need to be determined.

\section{References}

[1] Patrick Huber, "Neutrino Factory Sensitivity at Large $\theta_{13}$," presentation at the 7th IDSNF Plenary Meeting, October 17-19, 2011, Virginia Tech Research Center, Arlington, Virginia, slide 11. https://www.ids-nf.org/ wiki/VTECH-2011-10-17

[2] Sanjib K. Agarwalla, Patrick Huber, Jian Tang, and Walter Winter, "Optimization of the Neutrino Factory, Revisited," JHEP01 (2011) 120 , figure 5 , plots for $\sin ^{2} 2 \theta_{13}$ of 0.1 and 0.01 .
[3] The IDS-NF Collaboration, "Interim Design Report," IDS-NF-020. https://www.ids-nf .org/

[4] Gallardo, Juan C., "Note on the Calculation of Meson $(\pi, K)$ production distributions for different proton bunch length," NFMCCdoc-340. http://nfmcc-docdb.fnal.gov/ 